\title{
BioéthiqueOnline
}

\section{La bioéthique, l'art et le syndrome des ovaires polykystiques : propos impressionniste visant à réhabiliter les corps tabous et les ovaires blâmés}

\section{Victoria Doudenkova}

Volume 5, 2016

URI : https://id.erudit.org/iderudit/1044280ar

DOI : https://doi.org/10.7202/1044280ar

Aller au sommaire du numéro

Éditeur(s)

BioéthiqueOnline

ISSN

1923-2799 (numérique)

Découvrir la revue

Citer cet article

Doudenkova, V. (2016). La bioéthique, l'art et le syndrome des ovaires polykystiques : propos impressionniste visant à réhabiliter les corps tabous et les ovaires blâmés. BioéthiqueOnline, 5. https://doi.org/10.7202/1044280ar
Résumé de l'article

La bioéthique pousse à une prise de responsabilité de l'être humain face aux problèmes qu'il se crée tandis que l'art inspire un réenchantement dans le but de donner un sens différent au monde mécanique et technoscientifique d'aujourd'hui. La santé n'échappe pas à cette vision rigide et les croyances qui y sont reliées sont présentées dans un conte paradigmatique et absurde où la santé devient l'objet de la rationalité instrumentale et scientifique de la biomédecine. Suite à cette contextualisation, il devient plus aisé de comprendre le parcours frustrant décrit par les femmes atteintes du syndrome des ovaires polykystiques (SOPK) dans le contexte de leurs soins. La biomédecine conçoit le SOPK de façon biologique et individuelle oubliant l'apport d'une vision plus globale. À l'opposé de la biomédecine, des approches préventives cherchant l'amélioration de l'état de santé et non le traitement de la maladie, offrent une perspective plus holistique du soin. Dans ce contexte, l'art est conçu comme une façon originale de se souvenir des valeurs oubliées: une plus grande sensibilité et humanité, un rapport à la santé qui est respectueux de la vie, une créativité dans le contexte du soin. La démarche devient un geste moral et beau appelant non plus à lutter contre un mal, mais à prendre soin de la santé dans l'amour et la paix, ouvrant alors de nouvelles possibilités pour améliorer la qualité de vie des femmes concernées et donc produire un plus grand bien. 


\title{
La bioéthique, l'art et le syndrome des ovaires polykystiques: propos impressionniste visant à réhabiliter les corps tabous et les ovaires blâmés
}

\author{
ESSAI / ESSAY \\ Victoria Doudenkova ${ }^{1}$ \\ Reçu/Received: 19 Jan 2016 \\ Éditeurs/Editors: Vincent Couture \& Hazar Haidar \\ Travail créatif/Creative work: Stokes G. Constant Beauty Within and PCOS as Cacti. BioéthiqueOnline. 2016;5/21
}

2016 V Doudenkova, Creative Commons Attribution 4.0 International License

\section{Résumé}

La bioéthique pousse à une prise de responsabilité de l'être humain face aux problèmes qu'il se crée tandis que l'art inspire un réenchantement dans le but de donner un sens différent au monde mécanique et technoscientifique d'aujourd'hui. La santé n'échappe pas à cette vision rigide et les croyances qui y sont reliées sont présentées dans un conte paradigmatique et absurde où la santé devient l'objet de la rationalité instrumentale et scientifique de la biomédecine. Suite à cette contextualisation, il devient plus aisé de comprendre le parcours frustrant décrit par les femmes atteintes du syndrome des ovaires polykystiques (SOPK) dans le contexte de leurs soins. La biomédecine conçoit le SOPK de façon biologique et individuelle oubliant l'apport d'une vision plus globale. À l'opposé de la biomédecine, des approches préventives cherchant l'amélioration de l'état de santé et non le traitement de la maladie, offrent une perspective plus holistique du soin. Dans ce contexte, l'art est conçu comme une façon originale de se souvenir des valeurs oubliées: une plus grande sensibilité et humanité, un rapport à la santé qui est respectueux de la vie, une créativité dans le contexte du soin. La démarche devient un geste moral et beau appelant non plus à lutter contre un mal, mais à prendre soin de la santé dans l'amour et la paix, ouvrant alors de nouvelles possibilités pour améliorer la qualité de vie des femmes concernées et donc produire un plus grand bien.

\section{Mots clés}

syndrome des ovaires polykystiques, approche globale, responsabilité, éthique, art

\section{Summary}

Bioethics pushes human beings to confront and take responsibility for the problems they have created, while art inspires a re-enchantment in order to give a different meaning to what has become mechanical and technoscientific world. Health is not exempt from this rigid world view, and related beliefs are often presented in a paradigmatic and absurd fashion where health becomes the object of a biomedicine's instrumental and scientific rationality. Following this contextualization, it becomes easier to understand the frustrating journey described by women with polycystic ovary syndrome (PCOS) in the context of healthcare. Biomedicine conceives PCOS as biological and individual, neglecting the contribution of a more global perspective. In contrast to biomedicine, preventive approaches that seek to improve health status and not simply treat the disease offer a more holistic perspective of care. In this context, art is conceived as a unique way to remember forgotten values: a more humane and greater sensitivity, a relation to health that is respectful of life, a creativity in caring. The process becomes a moral and beautiful gesture that is not an appeal to fight against evil, but to take care of health with love and peace, while opening up new opportunities to improve the quality of life of these women and so produce a greater good.

\section{Keywords}

polycystic ovary syndrome, global approach, responsibility, ethics, art 
Affiliations des auteurs / Author Affiliations

${ }^{1}$ Programmes de bioéthique, Département de médecine sociale et préventive, École de santé publique (ÉSPUM), Université de Montréal, Montréal, Canada

\section{Correspondance / Correspondence}

Victoria Doudenkova, victoria.doudenkova@umontreal.ca

\section{Remerciements}

L'auteure tient à exprimer sa gratitude envers toutes les expériences enrichissantes qu'elle a vécue dans la vie, y compris celui qu'elle a eu l'occasion de vivre dans le cadre du projet Art + Bioéthique. Elle remercie Grâce Stokes pour la belle collaboration scientifico-artistique qu'elles ont développée ensemble. Elle tient également à remercier Jean-Christophe Bélisle Pipon, avec qui elle a été en mesure de partager et de nourrir ses pensées et ses idées au cours des derniers mois. Une mention spéciale à Vincent Couture pour sa gentillesse, son authenticité et pour toute l'ardeur qu'il a mise dans le processus éditorial. Les nombreux échanges entre lui et l'auteur, ainsi que son travail acharné a rendu possible le respect et la libre expression de la pensée de l'auteure dans le texte.

\section{Conflit d'intérêts}

Mme Doudenkova entretient une relation personnelle avec M. Bélisle-Pipon, l'un des éditeurs invités du dossier thématique. La situation a été gérée par le retrait de $M$. Bélisle-Pipon, lors de l'évaluation de la candidature de Mme Doudenkova ainsi que lors du processus éditorial et de la révision du présent texte.

\section{Acknowledgements}

The author wishes to express her gratitude to all the rewarding experiences she had in life, including the one she had the opportunity to live with Art + Bioéthique. She thanks Grace Stokes for the beautiful scientifico-artistic collaboration that they developed together. She also wishes to thank Jean-Christophe Bélisle Pipon, with whom she was able to share and nurture her thoughts and ideas over the past months. A special acknowledgment to Vincent Couture for his kindness, authenticity and for all the energy he has put into the editorial process. The many exchanges between him and the author, along with his hard work has made possible the respect and free expression of the author's thoughts in the text.

\section{Conflicts of Interest}

Ms. Doudenkova has a personal relationship with $\mathrm{Mr}$. Bélisle-Pipon, one of the guest editors of the thematic issue. The situation was handled by the withdrawal of $\mathrm{Mr}$. Bélisle-Pipon from assessing the candidacy of Ms. Doudenkova and during the editorial process and the revision of this text.

\section{Avant-propos}

Cet essai se propose d'utiliser l'opportunité offerte par Art + Bioéthique pour pousser plus loin l'hybridation de l'art et de la bioéthique. Notre proposition, différente, et qui sera potentiellement perçue comme étant polémique, souhaite poser un autre regard sur la santé et la maladie. Je reconnais que cette approche n'est pas sans limites. Plusieurs lecteurs resteront sur leur faim parce que je ne défendrai pas les idées proposées en m'appuyant sur la littérature scientifique. Beaucoup seront outrés, certains seront peut-être inspirés et c'est cela qui compte. J'emprunterai plutôt la voie de l'art et opterai pour un éloge de l'esthétique, des émotions, de la beauté et de la simplicité. Je concevrai l'éthique comme une notion prenant son sens dans le respect de la vie. Tout comme l'art explore, ici il s'agira de l'exploration d'idées dans la liberté non académique, dans la pensée non rationnelle. L'exercice est de faire le contraire de ce que je suis amenée à faire habituellement : une sorte de défi personnel, car il aurait été bien plus aisé pour moi de rédiger dans un style scientifique. L'objectif consiste donc à se heurter soi-même dans les conceptions et idées que l'on a admises sur la santé, la maladie et le monde. L'objectif est de rêver et d'explorer de nouveaux horizons, car l'art explore sans se soucier. L'art est libre et n'argumente pas. II ne cherche en rien à démontrer quelque chose. Cet essai se conçoit comme tel : c'est une expérience en soi qui reflète tant la réflexion, que l'émotion, l'imagination, le rêve, le lâcher-prise ainsi que différents états d'esprit qui ont spécifiquement été adoptés pour la rédaction des diverses parties du texte et qui ont grandement été inspirés par le Dr Bach. La production même fait partie de la démarche artistique. Dans une section, j'utiliserai la forme du conte pour montrer de façon caricaturale comment la biomédecine s'est détournée de la santé pour une culture de la maladie. Dans une autre, je parlerai du cas des femmes affectées par le syndrome des ovaires polykystiques, qui n'est pas encore une maladie, mais un syndrome montrant les signes d'une santé déjà affectée. Je terminerai par proposer la venue d'une science artistique comme façon de redonner une sensibilité éthique à la médecine et au soin, tout en revalorisant la place centrale de la personne affectée. 


\section{Introduction}

Reconnaître qu'en tant que société, certaines de nos actions contribuent à créer nos propres problèmes force de manière inverse à constater notre potentiel à pouvoir les régler à condition, bien sûr, de prendre en compte certaines de nos responsabilités. Un message d'espoir qui peut aider à limiter nos " fuites en avant ", qui servent justement à cautionner ou à diminuer les impacts de nos attitudes actuelles, et à regarder les causes réelles de nos maux. Voilà, à mon avis, le défi éthique d'aujourd'hui et de demain. Le défi de l'art, quant à lui, serait peut-être de contribuer au réenchantement du monde des humains et de révéler à leurs yeux la beauté et le sens qu'ils parviennent de plus en plus difficilement à y voir. C'est dans cette vision de complémentarité que l'artiste Grace Stokes et moi-même avons évolué dans le cadre de ce projet portant sur les enjeux éthiques entourant le soin des femmes atteintes du syndrome des ovaires polykystiques (SOPK).

Ce qui décevra et choquera potentiellement le scientifique réjouira sans doute l'artiste : la science force à considérer le monde par le biais de problèmes. Comme Karl Popper disait : " Le seul moyen d'accéder à la science, c'est de rencontrer un problème, d'être frappé par sa beauté, d'en tomber amoureux, de lui faire des enfants problèmes, de fonder une famille de problèmes. " [1] Puisqu'il ne sert à rien de perdre son énergie à combattre les failles, l'objectif de la démarche artistico-éthique amorcée dans le cadre de ce projet ne sera pas tant de dénoncer les problèmes de manière tout à fait scientifique, mais plutôt, en revendiquant la beauté et la simplicité comme principes directeurs, de valoriser ces failles. En effet, leur reconnaissance peut permettre d'encourager des prises de conscience là où le terrain est assez fertile pour les accueillir. II m'apparaît évident que l'art est tout indiqué pour une telle entreprise! En effet, parce qu'il s'agit là de bien plus qu'un simple support d'expression, l'art représente potentiellement l'un des moyens de communication les plus porteurs et complets qui existent. L'expression d'un artiste pouvant prendre toutes sortes de formes, n'est pas limitative comme peut l'être celle du véhicule phare dans le monde scientifique : l'article scientifique. Elle intègre des dimensions pouvant être multiples liées à l'esthétique et à l'émotion, qui résonnent avec l'entièreté de toute personne qui y est exposée, pour autant qu'elle y soit sensible. C'est pourquoi mettre en lien art et éthique permet de magnifier les enjeux discutés en proposant à chacun une perception résonnant avec sa sensibilité personnelle. L'art, contrairement à la science, a la faculté de toucher à des dimensions humaines dépassant celles uniquement cérébrales, comme la sensibilité, l'émotion ou encore la spiritualité. Mais l'éthique est de nature humaine et gagnerait à considérer ces dimensions dans la mesure où elle cherche souvent à sensibiliser. Alors, il serait possible d'arrêter de se battre contre les problèmes, ce qui n'a souvent pour effet que de les renforcer, et de se contenter de regarder les faits. II faudrait aussi limiter, dans la mesure du possible, les biais cognitifs et autres croyances que les psychologues nomment "dysfonctionnelles " visant à catégoriser les pensées stériles et stéréotypées. Faire cela permettrait de voir les lieux propices au changement positif et il serait alors possible de conscientiser la capacité que chacun a à pouvoir créer des solutions.

\section{Conte}

"Conventional medicine has failed to acknowledge the sad truth that man, along with other living creatures, will never be compatible with artificial chemicals, no matter how identical these chemicals may look, feel and smell compared to the natural varieties. " Carol Foster

Il était une fois une réalité où il était possible d'" attraper » une maladie, par hasard, par malchance. II y existait une sorte d'idéologie de la maladie avec tout un système prévu pour la gérer et la traiter. II était possible de déterminer les fonctions défaillantes et de trouver des moyens pour les remplacer. Grands étaient les progrès, surtout matériels! Toutefois, le matérialisme ambiant appelait à regarder l'objet, l'outil, la chose et non l'état d'esprit ou de conscience qui l'accompagne, posant dès lors la 
question : au service de quoi ces merveilles étaient-elles utilisées? À voir autant de corps défaillants partout, il a été aisé de croire que le corps humain fonctionnait mal. II fut alors facile de surenchérir dans une "lutte » contre ceci ou contre cela. Une lutte qui coutaît cher, dans tous les sens du terme. Pourtant, beaucoup fut élaboré à partir de cette croyance et l'on ne comptait plus nos efforts pour la perpétuer, car l'on considérait cela bon de se battre contre cet ennemi qu'est la maladie. Une lutte qui se traduisait toujours soit par des innovations technologiques de plus en plus complexes, soit par quelques préparations chimiques faisant tantôt du bien, tantôt du mal avec leurs effets secondaires. II est vrai, considérant le contexte dans lequel les gens étaient amenés à vivre, que ces préparations devenaient salutaires et même indispensables pour un grand nombre d'entre eux. Elles sauvaient et prolongeaient de nombreuses vies! Mais au bout du compte une chose importante restait ignorée : le combat des êtres humains se faisait avant tout contre eux-mêmes, et ce, dans un sentiment général de peur. Aussi, les malades étaient amenés à haïr leur corps défaillant et la responsabilité de la santé était léguée à toute autorité externe en mesure d'intervenir : le médecin qui devait guérir, la recherche qui devait décrire les maladies et créer le meilleur traitement, les industries qui proposaient avidement toujours plus à acheter ou encore d'autres institutions biopolitiques qui géraient, en somme, l'ensemble des questions de santé. Comme dès leur plus jeune âge les malades ont été conditionnés à rechercher des solutions extérieures à eux-mêmes pour régler leurs problèmes, ils étaient ravis de ces dernières, quand bien même il s'agissait souvent de pansements superficiels masquant des déséquilibres plus profonds. Bientôt ces déséquilibres furent oubliés eux aussi, car peu savaient quoi faire pour les régler, et il fut aisé de croire que la manifestation à elle seule constituait le problème. La médecine dite allopathique, alors monopole d'État, avait déclaré la guerre aux symptômes en amalgamant leur disparition ou leur contrôle à un retour à la santé, à une victoire. Cette médecine était douée pour sauver la vie, mais pas pour aider les malades à améliorer leur état de santé : la maladie chronique était devenue un fléau. II fallait faire taire toute manifestation, du bouton d'acné sur la figure à la masse cancéreuse dont le destin était de se faire violenter. La logique était guerrière, efficace, rapide. Peu importe si le problème finissait par revenir, il y avait toujours une autre arme à tester. Comme on fait taire l'enfant qui braille, on criait victoire devant un corps décrépi, sans symptômes, mais sans vie non plus. Le mot guérison fut remplacé par le mot rémission et la possibilité d'espérer plus était réservée à quelques chanceux ou rebelles. Chanceux étaient ceux avec un problème pouvant être guéri par un médicament ; rebelles, ceux ayant compris que, pour leur problème de nature plus chronique, ils étaient les seuls à pouvoir prendre leur santé en main pour éviter de passer leur vie à survivre en consommant des médicaments.

Finalement, plus grand-chose n'était su de la santé, dans quelles conditions il était possible de le rester ou de le redevenir. Des intérêts étant en jeu, beaucoup était fait afin de distraire les citoyens de la compréhension de leurs besoins réels. Le regard porté sur la physiologie était statique. Dans cette réalité, l'état de santé ne pouvait que se dégrader avec les années, les maladies devenaient chroniques, et le vieillissement fut l'une des plus grandes angoisses. Pour la mort, ce fut encore pire. Elle restait cachée à notre regard et parfois, à bout de souffle et de solutions, il fut même admis d'aider certains à mourir pour le soulagement de tous. Dans cette réalité, il fut oublié que la grande part des maladies vues comme étant une fatalité, étaient créées par l'être humain lui-même. Mais il fut surtout oublié, et c'est bien là le pire, que l'être humain aurait pu être capable d'entretenir ou de recréer les conditions propices au rétablissement de la santé, s'il en avait seulement eu l'intention. L'évidence qu'un corps en santé ne développait pas de maladies ne paraissait plus si évidente. L'investissement pour la santé fut tout simplement oublié aux dépens de l'investissement d'efforts et d'argent dans la maladie, et même si le traitement à vie de la maladie apportait un soulagement à de nombreuses personnes voire prolongeait leur vie, il restait privilégié, car plus lucratif que le premier et demandant moins de remise en question. Ainsi, les causes des maux chroniques accablant I'humanité étaient systématiquement ignorées ou rendues complexes, alors qu'elles étaient avant tout issues de la création humaine, faisant en sorte que le rétablissement n'était jamais envisagé comme une possibilité valable pouvant apporter encore davantage de bienfaits. Peut-être est-ce par là qu'il aurait fallu commencer dès lors que l'on cherchait à aider un malade? On ne savait finalement que si 
peu de choses sur la santé, qu'il s'agissait là d'une notion presque ésotérique, tandis que des maladies furent décrites par centaines.

Bien sûr, pour autant qu'ils en étaient conscients, certains voyaient dans ce conte une fiction aberrante. Tandis que d'autres y évoluaient, car c'était malheureusement leur réalité. Les femmes dont je vais vous parler ont été amenées à se confronter régulièrement à ce type de réalités, privilégiant la maladie plutôt que la santé. Elles, qui n'étaient pas encore malades, mais déjà en mauvaise santé, avaient le choix de suivre le sentier de la santé ou celui de la maladie. Bien qu'elles y voyaient parfois quelques espoirs, c'est surtout celui parsemé d'épreuves, de défis et de frustrations qu'elles semblaient emprunter.

\section{Le syndrome des ovaires polykystiques (SOPK)}

"We share a common experience in our refusal to be objects acted upon by the medical institution. Instead of tacitly following recommendations made by physicians or settling for diagnoses that do not make sense to us, we have claimed the right to ask questions and participate in active resistance that we believe will ultimately lead to better health outcomes. " Jennifer Lynn Ellerman

Le prunier mirobolant (cherry plum) est un arbre faisant partie de la famille des rosacées. On le retrouve dans le sud-est de l'Europe et en Asie occidentale. Sur la base de ses explorations intuitives, le Dr Edward Bach (1886-1936), médecin britannique, créa la thérapie par les élixirs floraux. II utilisa les magnifiques fleurs blanches du prunier dans un remède dit pour aider la peur de perdre le contrôle ou encore la peur de faire des choses épouvantables et redoutées [2]. La perte de contrôle redoutable est justement l'accusation principale qui est faite aujourd'hui, en toute discrétion, aux ovaires de plus en plus de femmes. Dès lors, ces petits organes d'espoir et de désespoir ont fini par refuser de remplir leur tâche mensuelle laissant des follicules immatures (les fameux kystes) s'y entasser anarchiquement par demi-douzaines. Ces 5 à $20 \%$ de femmes (selon les critères que l'on applique) sont atteintes de ce qui est appelé, potentiellement à tort (car les ovaires sont davantage victimes que coupables), le syndrome des ovaires polykystiques (SOPK) [3].

Véritable enjeu de santé publique, le SOPK est le désordre endocrinien le plus répandu chez la femme en âge de procréer [4]. Ayant un impact notable et reconnu sur la qualité de vie, il représente une des causes majeures d'infertilité et est associé à des conditions sérieuses comme les cancers hormono-dépendants (sein, utérus, ovaires), les maladies cardiovasculaires, le diabète de type 2, ou encore certains désordres psychologiques comme la dépression et l'anxiété. En raison de l'incapacité potentielle à porter des enfants, de l'hirsutisme (hyperandrogénie), de l'absence de menstruations, d'une fonction sexuelle perturbée, de l'obésité, ou encore de l'acné (les signes qui se manifestent sont propres aux déséquilibres métaboliques de chaque femme), les femmes sont à même de perdre leur sens de la féminité et d'éprouver honte et perte d'estime de soi face aux idéaux normatifs féminins (ex. : beauté, féminité et fertilité) [5]. II est dès lors d'usage pour les femmes de ne pas aborder ce sujet quelque peu tabou au sein du débat public, d'autant plus que l'espace qui y est laissé est aujourd'hui inversement proportionnel à la prévalence du désordre.

À la limite entre la santé et la maladie, il semble s'agir d'un problème féminin ignoré presque autant par les chercheurs (la recherche n'en est qu'à ses débuts et des lacunes importantes existent dans la compréhension de la physiopathologie du syndrome) que par la société, poussant dès lors le corps médical à œuvrer comme il le peut sans encore trop le comprendre. Dans son arsenal, quelques pilules permettent d'ignorer le problème à leur façon, en limitant les dégâts par le combat plus souvent du symptôme que de la cause - un combat perdu d'avance, mais qui peut suffire à occuper une vie. Grande reste encore aujourd'hui la déception des femmes face aux soins qui leur sont 
proposés. On peut citer à partir des rares études consacrées à l'expérience vécue des femmes sur le SOPK qui leurs donnent directement la parole: insensibilité et inattention de la part des professionnels de la santé, banalisation des symptômes, délais dans le diagnostic, accent et préoccupations majoritairement autour de la santé reproductive, manque de sensibilisation des médecins sur la condition et manque d'informations données [5-8]. Problème tant individuel que social et politique, car réaliser que certaines implications liées au SOPK peuvent être améliorées par un mode de vie plus adéquat, qu'il s'agisse de stress, de qualité de l'environnement (ex.: perturbateurs endocriniens) ou d'alimentation, force à questionner les modes de vie côtoyés quotidiennement au sein de notre société. Au vu de la prévalence grandissante du désordre, certaines femmes seraient a priori plus sensibles à ces modes de vie. Cela amène à remettre en question non plus seulement les causes individuelles, mais aussi sociales de cette condition dans une vision écoépidémiologique et défie quelque peu la tendance actuelle qui vise à ramener les facteurs de risque majoritairement à l'individu et à son génome [9].

En plaçant l'esprit humain au-dessus de toute chose et en admettant naïvement qu'il peut tout conceptualiser, qu'il peut tout réaliser, nous avons fini par ne voir le monde qu'à travers nos processus mentaux qui catégorisent tout, séparant les humains de la nature, les humains entre eux, l'individuel du collectif, le corps de l'esprit, les organes du corps, les fonctions physiologiques les unes des autres, la génétique de l'environnement, les symptômes de la cause et finalement la maladie du malade. En compartimentant ainsi le vivant, ces séparations ont rendu le monde si complexe, que parfois la jouissance mentale tirée à investiguer cette complexité jour après jour n'avait d'équivalent que notre tendance à ignorer le bon sens inné. Ainsi, la beauté intrinsèque au vivant et aux liens qui le caractérisent a été perdue. Dans ce contexte, il nous paraît aujourd'hui évident de croire que seuls des médicaments fabriqués par notre esprit soient capables de nous soigner. Postulat devenu indiscutable dans notre société, mais surtout si internalisé à tous les niveaux, que peu de place est laissée à toute autre conception. Par exemple, nous parlons de plus en plus de l'état écologique de la planète, mais nous ne parlons jamais (à l'exception de quelques pratiques de soins dites " alternatives ») de l'état écologique interne de notre corps, ce que l'on pourrait appeler l'endoécologie de la santé. II en va de même pour la médecine dite officielle dont l'influence " a connu une croissance exponentielle au cours du siècle dernier, l'institution médicale ayant consolidé sa position en tant qu'autorité dès lors que les questions liées à la santé sont concernées. Cette influence a été institutionnalisée à un tel point qu'elle est devenue omniprésente et rarement remise en question. " [10] (traduction libre) Discuter des problèmes féminins comme le SOPK apparaît être une porte intéressante pour questionner cette influence, d'autant plus qu'il s'agit là de systèmes de soin historiquement construits par l'homme blanc. L'intérêt de la démarche reste pertinent dans la mesure où, depuis l'appel féministe à une approche biopsychosociale de la santé [11] (sans justifier pour autant une légitimité à la médecine à s'instaurer comme autorité statutaire sur les dimensions supplémentaires qu'impliquent cette approche), l'évolution de notre manière de soigner reste encore minime.

Dans ce contexte, il est à se demander si à force de rechercher des solutions compliquées et dispendieuses que sont les traitements pharmacologiques ou autres approches technologiques, on manquerait à prendre au sérieux et à proposer des solutions simples et efficaces, ayant déjà fait leurs preuves millénaires (par exemple les plantes médicinales), tout en privilégiant l'action sur le mode de vie. Cela permettrait de laisser les autres solutions pour les cas où elles sont réellement nécessaires, si bien sûr elles ne sont pas devenues obsolètes. C'est ce que l'on commence timidement à recommander dans quelques écrits scientifiques traitant du SOPK [12]. Aussi, se détacher de l'idée qu'il faut nécessairement " soigner » le corps, permet de constater que parfois la seule chose qu'il est souhaitable de faire est d'arrêter de lui nuire en lui donnant les conditions nécessaires à sa régénération (faire moins au lieu de faire plus), concept malheureusement inexistant dans un modèle interventionniste. La régénération demande de l'attention, de la douceur, de l'engagement, de la patience et surtout de l'amour. À ce titre, en nous laissant inspirer par le Dr Bach, nous pouvons sortir de la logique qui vise à réparer le corps considéré comme une mécanique et nous ouvrir en toute 
simplicité à des visions différentes en regard de la santé. En ayant une sensibilité particulièrement développée, il comprenait l'impact des états émotionnels négatifs sur la santé, ces derniers affectant d'abord les champs énergétiques de l'individu pour ensuite se manifester dans le corps physique. II s'agirait, en s'inspirant de sa démarche, lors d'un soin moins contrôlant et plus collaboratif, d'avoir une approche qui considère la personne dans sa totalité (ex. : aspects spirituels, psychologiques, émotionnels, physiques, environnementaux).

En écho avec l'œuvre de Grace Stokes, c'est ce qu'investiguent certaines femmes ayant côtoyé les limites de l'approche symptomatique proposée en médecine (qui vise à mettre des pansements pour soulager les problèmes plutôt que d'essayer de les régler), en se tournant vers une logique de l'action qui promeut la santé contrairement à celle qui lutte indéfiniment contre la maladie dans un combat perdu d'avance. Dans un long processus d'essais et d'erreurs incluant tant un cheminement personnel que la compréhension approfondie de leurs maux qui révèlent les diverses possibilités de les soigner, elles rencontrent parfois à l'issue des bienfaits inespérés et comprennent alors davantage ce que veut dire être en meilleure santé. Propos pas si farfelu que cela, car quand on regarde attentivement la théorie relativement à l'étiologie du syndrome proposée par Tremellen et Pearce [13], on constate que le déséquilibre de la microflore intestinale avec l'état d'inflammation chronique qui en résulte pourrait être une pierre angulaire dans l'apparition du SOPK et donc une des causes possibles, car elle a le potentiel de l'expliquer à elle seule (en tous cas pour les phénotypes courants). À défaut, il serait un des morceaux centraux du casse-tête qui permettrait d'expliquer l'apparition du SOPK. II est dès lors aisé de concevoir la raison pour laquelle l'action sur le mode de vie est sujette à des résultats si positifs chez de nombreuses femmes. Elle rétablit la santé intestinale et conséquemment améliore la santé globale, rapprochant l'intestin des ovaires et défiant alors la vision séparée que l'on a du corps humain et de ses fonctions.

Pourtant, le problème est encore fortement vu comme étant essentiellement reproductif et, dès lors, l'attention est rapidement dirigée sur les organes possédant ladite fonction. II faut ajouter que celles bénéficiant de cette attention sont chanceuses, car seule une partie des femmes concernées seront mises au courant de leur état après un long périple médical et beaucoup ignoreront leur diagnostic, ou y feront face à un moment charnière d'une vie, quand l'aboutissement de la décision de devenir mère ne se manifestera tout simplement pas, l'infertilité brisant subitement les projets les plus précieux. Et là, dans l'élan de leur unique espoir, elles passeront directement d'une pilule à une autre, rentrant dans la " machine à saucisses » des traitements de fertilité (médicaments, forage ovarien, techniques de procréation assistée), en dépit des risques souvent plus grands pour ces femmes, I'horloge biologique de la reproduction imposant une contrainte d'âge qu'il faut savoir respecter si l'on ne veut pas manquer sa chance. Mais dans ce processus, bien qu'il soit légitime et parfois indispensable pour atteindre une fin tant désirée, amenant un espoir là où il n'y en avait plus, certaines questions méritent d'être posées. À force de mettre l'emphase sur la santé gynécologique de la femme, a-t-on oublié qu'il s'agit là encore d'une séparation essentiellement conceptuelle alors qu'il serait peut-être bien plus pertinent, pour ces femmes, de regarder le lien étroit qui existe entre l'état global de santé de la femme et sa fertilité? Avons-nous pris le temps de chercher à minimiser les conséquences relatives au fait de forcer un corps qui a déjà du mal à s'équilibrer lui-même (porteur de déséquilibres endocriniens et métaboliques) à porter un enfant? Comment pourrions-nous aider ces femmes à avoir une grossesse sereine, qu'elles aient besoin de traitements ou non? L'autonomie reproductive est-elle celle qui nous permet de choisir par quelle " machine à saucisse » nous souhaitons passer et quand, ou serait-ce plutôt de prendre conscience du rôle actif que nous pouvons jouer quand nous souhaitons que nos corps affectés et fatigués créent la vie (sans pour autant exclure une aide technologique éventuelle si elle s'avère indispensable)? Et là, quelle place est donnée au respect de l'autonomie dans le choix parmi les options de soins qui existent réellement et non uniquement celles d'office présélectionnées par une culture ou une société (ex. : médicaments, technologies procréatives) qui ne sont pas forcément les plus sécuritaires ni les plus efficaces pour une grande partie des femmes affectées par le SOPK (ex. : les femmes obèses)? La lutte pour ce que 
l'on souhaite est un objectif louable, mais quelle place laissons-nous à la collaboration avec nousmêmes dans l'amour et l'empathie?

\section{D'abord, ne pas nuire}

"L'éthique, c'est la reconnaissance de notre responsabilité envers tout ce qui vit. " Albert Schweitzer

Tout comme la paix n'est pas l'absence de guerre, mais plutôt la présence de justice, la santé n'est pas l'absence de maladie, mais peut-être la présence du « meilleur état possible d'équilibre dynamique physique, psychique et socioculturel de l'organisme dans son environnement écologique. » [14] L'être humain ne cesse de lutter pour telle ou telle chose. La lutte est utile dans l'urgence ou dans les circonstances exceptionnelles. On lutte pour survivre, avec agressivité et ardeur. C'est une force dont une société a besoin en certaines circonstances seulement et non comme mode premier d'action. Lutter contre la maladie, dans un état d'esprit totalitaire où nous imposons nos volontés au corps sans prendre en compte ses besoins les plus élémentaires, nous fait oublier que la santé s'établit avant tout dans une attention bienveillante envers ce corps qui est notre plus grand partenaire de vie. La santé n'est pas un bien qui est là " par défaut » et il faut en prendre soin au quotidien. La lutte est issue de la peur, et la peur de la séparation créée par nos processus mentaux visant à tout catégoriser. La santé, elle, ne peut qu'émerger de l'amour et de la solidarité. Elle appelle à l'harmonie et à l'équilibre, à la douceur, à un soin attentionné et humain, à la relation d'aide et à la collaboration, ou encore au respect du corps, de son environnement, de ses besoins et de ses processus physiologiques. Bref, elle fait appel au respect de la vie. Vue ainsi, la santé ne peut être dissociée de ce que l'on appelle l'éthique.

Parfois, il pourrait être utile d'aller au-delà du statu quo, des peurs et du conditionnement afin de porter un regard lucide sur nos malaises, qu'ils soient humains, sociaux ou environnementaux. II est possible que ce soit là que résiderait l'espoir de trouver certaines solutions à nos problèmes de plus en plus nombreux et " complexes ». Célébrer la beauté et la collaboration au lieu de la séparation et de la violence, nous éveillerait peut-être davantage aux potentialités du vivant. Et nos ovaires, ne craignant plus de perdre le contrôle de leur fonction, pourraient enfin retrouver cet équilibre qui leur permettrait de guérir et, qui sait, de créer un jour la vie.

\section{Science artistique}

" Any intelligent fool can make things bigger, more complex and more violent. It takes a touch of genius and a lot of courage to move in the opposite direction." " Albert Einstein

II semble aujourd'hui que les élixirs floraux du Dr Bach n'ont rien de scientifique et sans doute il continuera de passer pour un charlatan, car la science étudie les choses de l'esprit, définissables, déterminées et rationnelles, tombant dans des catégories précises et rassurantes par leur stabilité. Légitime, mais partielle, en créant une réalité qu'elle s'autorise à étudier, la science ignore cependant celle du Dr Bach. Elle était emplie de spontanéité, d'imagination, de ressenti, d'intuition, d'empathie, de créativité plus artistique que scientifique sans pour autant en perdre sa rigueur, une rigueur certes différente, mais qui l'a suivie tout au long de ses années, pour ne dire autrement...de recherche! C'est donc un peu de cette démarche vivante et artistique - on peut y voir la contribution majeure que peut apporter l'artistique au scientifique (démarche qui a directement inspiré la rédaction de l'essai) qui pourrait notamment aider à considérer de nouveau l'ensemble des médecines comme un art et nuancer le besoin excessif de contrôle que l'acquisition scientifique de données et d'évidences nous pousse à avoir. 
Par la recherche de la sécurité et de la protection à tout prix, c'est l'humanité et l'humilité qui, en cours de route, ont été perdues. Faible est l'étonnement quand les médecins deviennent les plus malades et les chercheurs les plus perdus, ou encore quand on ne reconnaît plus que par sa formule chimique le parfum d'une fleur. Nos productions intellectuelles, bien que vitales, nous détachent et nous emprisonnent toujours un peu plus de ce qui nous entoure. En s'enfermant dans les séparations ainsi créées, l'être humain est en train de perdre à son propre jeu et il ne pourra en sortir qu'à la condition de retrouver enfin la capacité de ressentir à nouveau son monde, retrouvant enfin un équilibre entre sa tête, sa conscience et son cœur. Plus que jamais, un regard nouveau est nécessaire sur nos réalités trop longtemps construites par les intérêts dominants. Plus que jamais, un regard artistique et créatif pourrait nous aider, non plus à réduire et à classifier notre réalité devenue objective et complexe, mais au contraire à savourer avec sensibilité les parfums variés et uniques de l'existence.

\section{Références}

1. Popper K. Le réalisme et la science. Hermann. Paris; 1990.

2. Blome G. Advanced Bach Flower Therapy: A Scientific Approach to Diagnosis and Treatment. Inner Traditions / Bear \& Co; 1999. 406 p.

3. Teede H, Deeks A, Moran L. Polycystic ovary syndrome: a complex condition with psychological, reproductive and metabolic manifestations that impacts on health across the lifespan. BMC Med. 2010;30;8(1):41.

4. Carmina E, Lobo RA. Polycystic ovary syndrome (PCOS): arguably the most common endocrinopathy is associated with significant morbidity in women. $\mathrm{J}$ Clin Endocrinol Metab. 1999;84(6):1897-9.

5. Kitzinger C, Willmott J. 'The thief of womanhood': women's experience of polycystic ovarian syndrome. Soc Sci Med. 2002;54(3):349-61.

6. Crete J, Adamshick P. Managing polycystic ovary syndrome: what our patients are telling us. J Holist Nurs. 2011;1;29(4):256-66.

7. Weiss TR, Bulmer SM. Young women's experiences living with polycystic ovary syndrome. J Obstet Gynecol Neonatal Nurs. 2011;40(6):709-18.

8. Williams S, Sheffield D, Knibb RC. 'Everything's from the inside out with PCOS': exploring women's experiences of living with polycystic ovary syndrome and co-morbidities through Skype interviews. Health Psychol Open. 2015;2(2). Doi:10.1177/2055102915603051

9. Leclerc B-S. L'épidémiologie contemporaine en crise de paradigme. Ruptures Rev Transdiscipl En Santé. 2005;10(2):178-98.

10. Ellerman JL. Don't Blame It on My Ovaries: Exploring the Lived Experience of Women with Polycystic Ovarian Syndrome and the Creation of Discourse. 2012. MA Thesis; Womens Studies. University of South Florida.

11. Weisman CS. Changing definitions of women's health: implications for health care and policy. Matern Child Health J. 1997;1(3):179-89.

12. Dumesic DA, Oberfield SE, Stener-Victorin E, Marshall JC, Laven JS, Legro RS. Scientific statement on the diagnostic criteria, epidemiology, pathophysiology, and molecular genetics of polycystic ovary syndrome. Endocr Rev. 2015;36(5):487-525.

13. Tremellen K, Pearce K. Dysbiosis of gut microbiota (DOGMA) - A novel theory for the development of polycystic ovarian syndrome. Med Hypotheses. 2012;79(1):104-12.

14. Donadieu Y. Ma pharmacie naturelle: Les meilleures thérapeutiques douces pour votre santé au quotidien. R. Laffont; 2008. 686 p. 